\title{
BOX-BEHNKEN DESIGN: A STATISTICAL APPROACH TO EVALUATE THE EFFECT OF CROSSLINKED CARBOXYMETHYL CELLULOSE AND SODIUM STARCH GLYCOLATE ON RELEASE KINETICS OF DRUG
}

\author{
GHULAM ABBAS ${ }^{1,2}$, MUHAMMAD HANIF'*, AKHTAR RASUL 2 , SAJID MAHMOOD KHAN ${ }^{3}$, \\ MUHAMMAD NAEEM AMIR ${ }^{2}$, BUSHRA NASIR ${ }^{1}$ and MUBASHAR AZIZ ${ }^{4}$ \\ ${ }^{1}$ Faculty of Pharmacy Bahauddin Zakariya University Multan, Pakistan \\ ${ }^{2}$ Faculty of Pharmaceutical Sciences Government College University Faisalabad, Pakistan \\ ${ }^{3}$ Faculty of Pharmacy and Alternative Medicines The Islamia University Bahawalpur, Pakistan \\ ${ }^{4}$ Department of Pathobiology, Faculty of Veterinary Sciences Bahauddin Zakariya University Multan, \\ Pakistan
}

\begin{abstract}
The aim of the study was to evaluate the release kinetics of domperidone maleate (DM) from immediate release (IR) tablets prepared by wet granulation method. Box-Behnken design (BBD) was used to optimize and evaluate the main, interaction and quadratic effects of independent variables i.e. crosslinked carboxymethyl cellulose $(\mathrm{CMC})\left(\mathrm{X}_{1}\right)$, sodium starch glycolate $(\mathrm{SSG})\left(\mathrm{X}_{2}\right)$ and starch $\left(\mathrm{X}_{3}\right)$ on responses $R^{2}$ of first order $\left(\mathrm{Y}_{\mathrm{I}}\right)$ and $\beta$ value of Weibull model $\left(\mathrm{Y}_{2}\right)$. Prepared tablets were characterized by various physical tests, invitro drug release, Fourier transform infrared spectroscopy (FTIR), X-ray diffractometry (XRD) and differential scanning calorimetry (DSC). Accelerated stability studies were performed on optimized formulation D9. $\mathrm{Y}_{1}$ and $\mathrm{Y}_{2}$ were ranged from 0.9959 to 0.9994 and 0.041 to 0.912 respectively. $\beta$ value of Weibull model indicated the parabolic shape of the dissolution curve. The quadratic model fits the data well and the resulting equations were used to predict the responses in the Box-Behnken design. FTIR spectra showed the compatibility of DM with CMC and SSG. XRD presented diffraction lines indicates crystalline nature of the drug. DSC thermograms indicated endothermic peak at $220^{\circ} \mathrm{C}$ for DM. Stabilities studies revealed that no significant change in hardness, friability, disintegration time and dissolution release profile of DM. It is concluded that a combination of CMC and SSG can be used to enhance the dissolution and release kinetics of IR tablets of DM.
\end{abstract}

Keywords: domperidone maleate, drug release kinetics, FTIR, DSC, XRD, quality by design

DM (6-chloro-3-[1-[3-(2-oxo-3H-benzimidazol-1-yl) propyl] piperidin]-1H-benzimidazol-2one), a powerful prokinetic drug, regulate the peristaltic movement of gastrointestinal tract by acting on dopamine receptors as antagonist. DM rarely cause extrapyramidal side effects because it does not cross the blood-brain barrier. DM is used in the treatment of motility disorders, nausea associated with chemotherapeutic agents, gastroesophageal reflux disorders and parkinson's disease $(1,2)$.

The oral route of drug administration is the most preferred and convenient way for treatment of any diseased condition. In this context, tablets are the most preferred and suitable dosage form. This dosage form drags the attention of different researchers due to their chemical and physical stability, accurate dosage, and easy way of releasing the drug. These dosage forms are acquiring a good place in delivery of drugs because they help in releasing the drug rapidly and improve the therapeutic effectiveness of the drug inside the body.

Superdisintegrants are extensively used in IR tablet formulations that support the dispersion and result in the release of drugs. CMC (3) and SSG are most popular superdisintegrants. Disintegration of orally administered tablets normally takes place in the gastrointestinal mucosa. There is no single mechanism which can explain the disintegration behavior of CMC and SSG. The main suggested mechanisms of disintegration include swelling, deformation recovery, and liquid wicking. Both CMC and SSG absorb a large quantity of water and promote rapid disintegration of tablets. Swelling is the most important mechanism for CMC and SSG.

* Corresponding author: e-mail: muhammad.hanif@bzu.edu.pk 
SSG is hygroscopic, absorb water, swell and show rapid burst effect. In this article, CMC and SSG are used first time in combined form to study the release kinetics of DM from IR tablets (4).

Olcer et al., in 2014 prepared the immediate release tablets of perindopril by direct compression technique and studied the in vitro drug release pattern and assay of active ingredients (5). Elkhodairy et al., in 2014 studied the behavior of SSG on the release profile of flutamide from fast dissolving tablets by using response surface methodology (6). In 2009 Late and Banga studied the effect of superdisintegrants on fast dissolving tablets by using response surface methodology (7). There has been no research paper published covering the IR formulation of DM and their characterization.

Drug release models have been used mainly to predict the release of drugs from different dosage forms. These models add value in terms of confirming the optimum design of pharmaceutical formulations as well as to comprehend the release mechanism of drugs through experimental authentication (8). Release kinetic models are applied on drug release mechanism from different dosage forms such as tablets, capsules, microspheres and nanospheres etc. First order and Weibull model are useful to study the release kinetics from IR formulations (9). In this article, response surface methodology is applied on release kinetics of IR tablet formulations of DM.

In this study, response surface methodology (7) was used to optimize 17 formulations of IR tablets as it provides main, interaction and quadratic effects using CMC and SSG as superdisintegrants. Experiments were performed using three-factor, three-level response surface Box-Behnken design. This design is appropriate for exploring quadratic response surfaces and it permits development of a polynomial model. Drug release models such as first order release and weibull models were used to predict the dissolution behavior and release kinetics of prepared tablets. Assay of the prepared tablets were performed by HPLC. The prepared tablets were further characterized by FTIR, XRD and DSC. Stability studies were performed on optimized IR tablets for a period of six months.

\section{EXPERIMENTAL}

\section{Materials}

DM was obtained as a gift sample form Shrooq Pharmaceuticals Pvt. Ltd. Lahore, Pakistan. Acetonitrile, CMC, and SSG were purchased from SigmaAldrich Chemie Gmbh, Germany. Potassium dihydrogen phosphate, starch, magnesium stearate and lactose were obtained from Darmstadt, Germany. All other reagents/chemicals used were of analytical grade.

\section{Experimental design}

Box-Behnken response surface designs are used to require three levels, coded as $-1,0$, and +1 (10). BBD was used for optimization of IR tablets of DM having 3 independent variables and 2 dependent variables using design expert (version 7.1 state-ease Inc., Minneapolis, MN). Independent variables were percentages of CMC $\left(X_{1}\right)$, SSG $\left(X_{2}\right)$ and starch $\left(X_{3}\right)$ while the dependent variables were $R^{2}$ of first order release model $\left(\mathrm{Y}_{1}\right), \beta$ of Weibull model $\left(\mathrm{Y}_{2}\right)$ as shown in Table 1. Many trials were performed for the selection of percentage of independent variables. On the basis of these trials, the design expert was used to generate the representative combinations of three independent variables at three levels and the entire design consisted of 17 experimental runs as shown in Table 2. The quadratic model by this design is given as:

$$
\begin{aligned}
Y i=b_{0} & +b_{1} X_{1}+b_{2} X_{2}+b_{3} X_{3}+b_{12} X_{1} X_{2}+b_{13} X_{1} X_{3} \\
& +b_{23} X_{2} X_{3}+b_{11} X_{1}^{2}+b_{22} X_{2}^{2}+b_{33} X_{3}^{2}
\end{aligned}
$$

Where $\mathrm{Yi}$ is the measured response of the dependent variables, $b_{0}$ is the intercept, $b_{1}$ to $b_{33}$ are the regres-

Table 1. Variables and constrains in Box-Behnken design.

\begin{tabular}{|l|c|c|c|c|}
\hline \multicolumn{1}{|c|}{ Variables } & \multicolumn{3}{c|}{ Level } & \multirow{2}{*}{ Constrains } \\
\cline { 1 - 4 } Independent & -1 & 0 & +1 & \\
\hline $\mathrm{X}_{1}$ CMC (\%) & 1 & 2.5 & 4 & In the range \\
\hline $\mathrm{X}_{2}$ SSG (\%) & 1 & 2 & 3 & In the range \\
\hline $\mathrm{X}_{3}$ Starch (\%) & 15 & 22.5 & 30 & In the range \\
\hline Dependent & & & & \\
\hline $\mathrm{Y}_{1} \mathrm{R}^{2}$ value of first order kinetic & & & & 0.99 to 1 \\
\hline $\mathrm{Y}_{2} \beta$ value of Weibull model & & & & Less than 1 \\
\hline
\end{tabular}


Table 2. Observed responses for immediate release tablets.

\begin{tabular}{|c|c|c|c|c|c|}
\hline \multirow{2}{*}{ Formulation } & \multicolumn{3}{|c|}{ Independent variables } & \multicolumn{2}{c|}{ Dependent variables } \\
\cline { 2 - 6 } & $\mathrm{X}_{1}(\%)$ & $\mathrm{X}_{2}(\%)$ & $\mathrm{X}_{3}(\%)$ & $\mathrm{Y}_{1}$ & 0.9991 \\
\hline D1 & 2.5 & 2 & 22.5 & 0.9989 & 0.096 \\
\hline D2 & 2.5 & 2 & 22.5 & 0.9993 & 0.195 \\
\hline D3 & 2.5 & 1 & 15 & 0.9992 & 0.077 \\
\hline D4 & 4 & 3 & 22.5 & 0.9991 & 0.050 \\
\hline D5 & 4 & 2 & 15 & 0.9985 & 0.912 \\
\hline D6 & 1 & 3 & 22.5 & 0.9989 & 0.092 \\
\hline D7 & 2.5 & 2 & 22.5 & 0.9991 & 0.177 \\
\hline D8 & 2.5 & 2 & 22.5 & 0.9994 & 0.129 \\
\hline D9 & 2.5 & 3 & 15 & 0.9989 & 0.041 \\
\hline D10 & 4 & 2 & 30 & 0.9897 & 0.486 \\
\hline D11 & 1 & 1 & 22.5 & 0.9989 & 0.102 \\
\hline D12 & 2.5 & 2 & 22.5 & 0.9992 & 0.133 \\
\hline D13 & 4 & 1 & 22.5 & 0.9959 & 0.721 \\
\hline D14 & 2.5 & 1 & 30 & 0.9984 & 0.041 \\
\hline D15 & 1 & 2 & 15 & 0.9992 & 0.166 \\
\hline D16 & 1 & 2 & 30 & 0.9990 & 0.103 \\
\hline D17 & 2.5 & 3 & 30 & & \\
\hline
\end{tabular}

sion coefficients computed from the observed experimental values of $\mathrm{Y} . \mathrm{X}_{1}, \mathrm{X}_{2}$, and $\mathrm{X}_{3}$ are the coded value of the independent variables. $X_{a} X_{b}(a, b=1$, $2,3)$ and $X_{i}^{2}(i=1,2,3)$ represent the interaction and quadratic terms, respectively.

\section{Experimental design validation}

The experimental values were compared with predicted values and percent relative error was calculated using this equation.

Percent relative error $=\frac{\text { Predicted value }- \text { Experimental value }}{\text { Predicted value }} \times 100$ (2)

\section{Preparation of IR tablets}

Tablets were prepared by mixing DM, CMC, SSG, starch, lactose and magnesium stearate by using sigma mixer and passed through a 20-mesh screen (11). Powder blend passed from the micromeritic limits were mixed thoroughly for 5 min by using sigma mixer Erweka Gmbh Heusenstramm Germany, capacity $5 \mathrm{~kg}$, model 53296. The mixture was granulated using $2 \%(\mathrm{w} / \mathrm{w})$ HPMC E5 in a $90 \%$ ethanol solution. $2 \%(w / w)$ HPMC E5 in 90\% ethanol used as granulating agent. The prepared granules were dried at $40^{\circ} \mathrm{C}$ for $2 \mathrm{~h}$, passed through an 18-mesh screen. Granules were compressed by using Minipress MII (Pharma Test, Hainburg, Germany).

\section{Pre-compression evaluation}

The angle of repose of powder blends and granules was measured by funnel method. Accurately weighed powder and granules were passed through the funnel. Diameter of the cone of powder and granules were measured and angle of repose was calculated using equation 3. Bulk and tapped density of powder and granules were calculated by cylindrical method. From each formulation $2 \mathrm{~g}$ of powder or granule samples were taken and introduced in $10 \mathrm{~mL}$ cylinder and initial volume was determined. Carr's index and Hausner's ratio were calculated by using the values of bulk and tapped densities. The determinations were carried out in triplicate form.

$$
\begin{gathered}
\tan \theta=\frac{h}{r} \\
\text { Bulk density }=\frac{M}{V_{b}} \\
\text { Tapped density }=\frac{M}{V_{t}} \\
\text { Carr's index }=\frac{V_{b}-V_{t}}{V_{b}} \times 100 \\
\text { Hausner's Ratio }=\frac{P_{t}}{P_{d}}
\end{gathered}
$$

Where $\theta$ is angle of repose, $\mathrm{h}$ is height of cone, and $r$ is radius of the cone base, $M$ is mass, $V_{b}$ is bulk volume, and $\mathrm{V}_{\mathrm{t}}$ is tapped volume, $\mathrm{P}_{\mathrm{t}}$ is tapped density, and $\mathrm{P}_{\mathrm{d}}$ is bulk density. 
Post-compression evaluation

Thickness, diameter, hardness, and weight variation

Thickness and diameter of prepared tablets were measured by placing the tablet in two arms of digital vernier caliper (Homeland China, model 4300121). Hardness was measured by Erweka hardness tester (Erweka 63150 Heusenstramm, Germany) and expressed in terms of $\mathrm{kg} / \mathrm{cm}^{2}$. Uniformity of weight was carried out according to European Pharmacopoeia (EP $4^{\text {th }}$ edition) specifications. From each formulation 20 tablets were taken, weighed individually, calculating the average weight and compared the individual weight of the tablet to the average weight. Five measurements were taken and percent relative standard deviation was calculated.

\section{Friability and disintegration time}

Friability of tablets were determined according to EP specifications. Twenty tablets from each formulation were taken in drum of Roche friability tester (Erweka D-63150 Heusenstramm, Germany). The drum was rotated for $4 \mathrm{~min}$ at the rate of 25 rpm, tablets were removed, dedusted and weighed accurately. The percent weight loss was calculated by equation 8 . Five measurements were taken for each batch.

$$
\text { Friability }(\%)=\frac{W_{\text {initial }}-W_{\text {final }}}{W_{\text {initial }}} \times 100
$$

Disintegration time of tablets $(n=6)$ was measured by compendial disintegration test apparatus (Erweka, DT, Germany) according to United State Pharmacopoeia 32. Distilled water was used as medium and temperature was at $37{ }^{\circ} \mathrm{C}$. Basket was oscillated at a constant speed of 30 cycles per min and randomly select six tablets from each formulation.

\section{In vitro drug release studies and release kinetics}

The in vitro release of DM was carried out in $900 \mathrm{~mL} \mathrm{SGF}$ of $\mathrm{pH} 1.2$ at $37 \pm 0.5^{\circ} \mathrm{C}$ from 0 to 60 min by using USP dissolution apparatus II at 100 rpm. $5 \mathrm{~mL}$ aliquot was pipette out at 1, 2, 3, 4, 5, $10,15,20,25,30$ and $60 \mathrm{~min}$ intervals and replaced with fresh medium of the same volume. The aliquot was filtered by 0.45 um filter and concentration of DM was determined by UV spectrophotometer (PerkinElmer Inc. New York, USA) at $285 \mathrm{~nm}$. The mechanisms of IR tablet formulations were determined by different in-vitro kinetics models such as first order (equation 9) and Weibull model (equation 10).

$$
\ln (1-F)=-K_{1} t
$$

$$
m=\exp \left|\frac{-\left(t-T_{i}\right)^{\beta}}{\alpha}\right|
$$

Where $\mathrm{F}$ is fraction of drug release in time $t, \mathrm{~K}_{1}$ is first order release constant, $\mathrm{m}$ is drug fraction accumulated in the solution on time $t, \alpha$ is the timescale process, $T_{i}$ is the latency time of the release process and $\beta$ is characterizes the type of curve. When $\beta$ value is 1 it indicates exponential shape, greater than 1 indicates sigmoid shape and less than 1 indicate parabolic shape.

\section{Assay}

The concentration of DM in IR tablets were analyzed according to BP Pharmacopoeia specifications using an HPLC system (PerkinElmer 710 Bridgeport Avenue Shelton, Connecticut USA) fitted out with RHS $\mathrm{C}_{18}$ column $(1.5 \mathrm{~cm} \times 4.6 \mathrm{~mm}, 5$ $\mu \mathrm{m})$ Agilent Technologies Santa Clara, California, United States. Twenty tablets were taken and grounded into fine powder. An amount equal to average tablet weight was transferred to a conical flask and add mobile phase to dissolve the active material. The solution was sonicated for $10 \mathrm{~min}$ for complete dissolution of active material. The analysis was performed at room temperature using a mobile phase consisting of acetonitrile and phosphate buffer $\mathrm{pH} 4$ in ratio of $1: 1$ at a flow rate of $1 \mathrm{~mL} / \mathrm{min}$. The detection wavelength was set at $285 \mathrm{~nm}$. The method was validated according to $\mathrm{ICH}$ guidelines. Linearity, specificity, recovery, precision and robustness parameters were performed. The retention time od DM was $6.298 \pm 0.5 \mathrm{~min}$. The linearity parameter was studied in the range of 3.125 to 25 ppm with a correlation coefficient $\left(R^{2}\right)$ of 0.999 . Percentage recovery was calculated as $100.5 \%$. The percent relative standard deviation of precision value was found less than $2 \%$.

\section{Fourier transform infrared (FTIR) spectroscopy}

The DM, superdisintegrants, and tablets of the optimized formulation were crushed with pestle and motor and dried at $40^{\circ} \mathrm{C}$. The samples were directly analyzed by using attenuated FTIR. The FTIR spectra over the wavelength range $800-3500 \mathrm{~cm}^{-1}$ have been recorded by FTIR spectrophotometer (Bruker Alpha, Germany).

\section{X-ray diffractometry (XRD)}

XRD diffractometer D/max-2500pc, Rigaku Co, Japan was used to obtain the diffractograms of drug, superdisintegrants and tablet of optimized formulation D9. Tube voltage was $40 \mathrm{kV}$, current was $\mathrm{mA}$ and scanning was $5^{\circ}$ over a range of 80-800 diffraction angle. 


\section{Differential scanning calorimetry (DSC)}

Thermal behavior of drug, superdisintegrants, and tablet of optimized formulation D9 were studied by differential scanning calorimeter DSC-60 Shimadzu, Germany. $5.5 \mathrm{mg}$ sample was placed in aluminum pans, sealed and analyzed under a stream of nitrogen gas of $100 \mathrm{~mL} / \mathrm{min}$ and heated from 50 to $350^{\circ} \mathrm{C}$.

\section{Stability studies and statistical analysis}

Stability studies were carried out according to ICH guidelines for six months by storing the optimized formulation in stability chamber at $40^{\circ} \mathrm{C}$ temperature and $75 \pm 5 \%$ relative humidity. Stability samples of the D9 formulation were evaluated for hardness, friability, disintegration time and dissolution release profile after one, three and 6 months. The data was expressed as mean descriptive statistics and one-way ANOVA was used for data analysis.

\section{RESULTS AND DISCUSSION}

\section{Experimental design, statistical analysis and opti- mization}

Interaction between factors and responses were studied and three-dimensional plots were developed

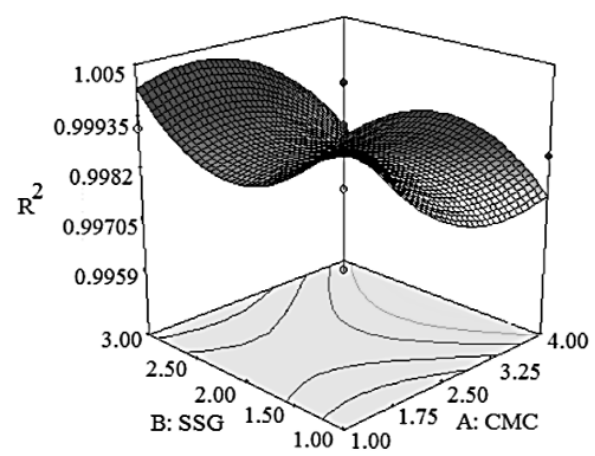

A

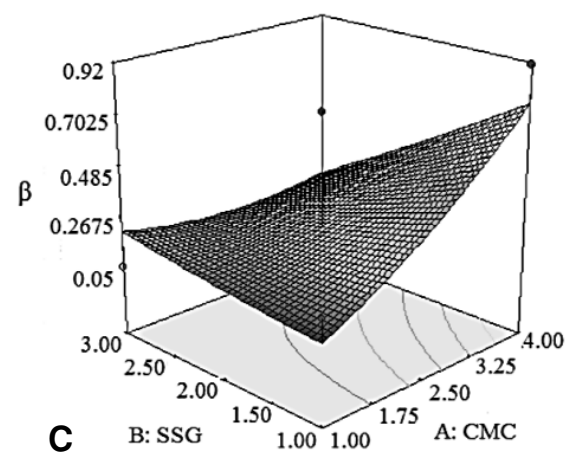

as shown in Figure 1. Values of $R^{2}$ of the first order release kinetic model were ranged from 0.9959 to 0.9994. $\beta$ values of Weibull model were ranged from 0.041 to 0.912 as shown in Table 2. Values of $R^{2}$ and $\beta$ were fitted to the quadratic models using BBD. The amount of CMC and SSG have a positive effect on disintegration time and release kinetics of DM. The relationship among three independent variables are non-linear quadratic and this is shown in response surface graph. Data of $Y_{1}$ and $Y_{2}$ were observed and the best-fitted model was quadratic and regression equations 10 and 11 were generated.

$\mathrm{Y}_{1}=1.00-1.200 \mathrm{E}-003 \mathrm{X}_{1}-1.250 \mathrm{E}-005 \mathrm{X}_{2}+$

$1.1378 \mathrm{E}-004 \mathrm{X}_{3}+1.500 \mathrm{E}-004 \mathrm{X}_{1} \mathrm{X}_{2}+2.200 \mathrm{E}-$

$003 \mathrm{X}_{1} \mathrm{X}_{3}+7.500 \mathrm{E}-005 \mathrm{X}_{2} \mathrm{X}_{3}-1.073 \mathrm{E}-003 \mathrm{X}_{1}^{2}+$

$$
1.502 \mathrm{E}-003 \mathrm{X}_{2}^{2}-9.475 \mathrm{E}-004 \mathrm{X}_{2}^{3}
$$

$\mathrm{Y}_{1}=0.25+0.16 \mathrm{X}_{1}-0.11 \mathrm{X}_{2}-0.061 \mathrm{X}_{3}-0.017 \mathrm{X}_{1} \mathrm{X}_{2}$

$-0.096 X_{1} X_{3}-0.010 X_{2} X_{3}+0.052 X_{1}^{2}+0.011 X_{2}^{2}$

$-\quad 0.14 \mathrm{X}_{2}^{3}$

\section{$R^{2}$ of first order release}

Positive signs of equation 11 showed optimization while negative signs revealed an inverse relationship between factors and responses. The model was found to be significant $(F$-value $=2.14 ; p<$ 0.001). The values for predicted (0.664) and adjust-

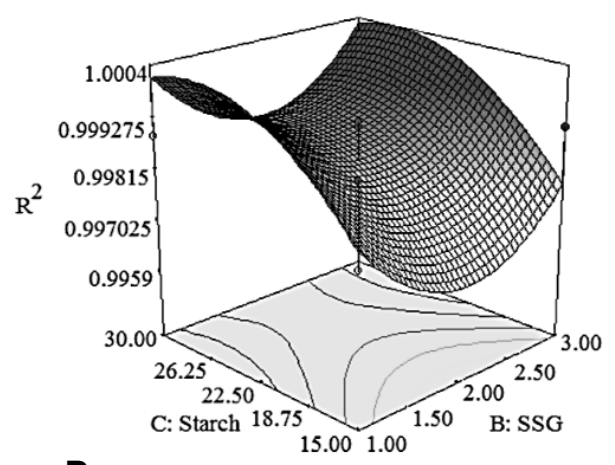

B

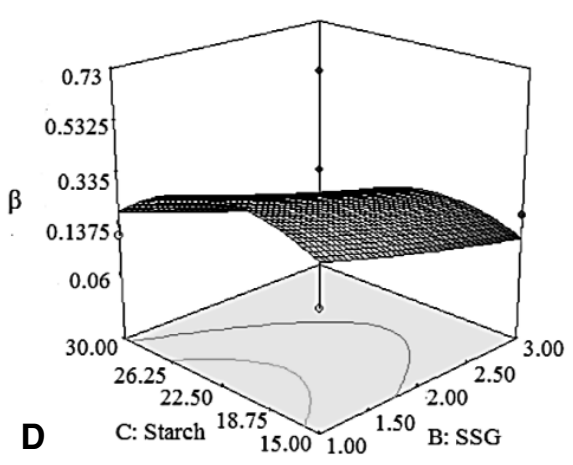

Figure 1. Response surface plots (A, B, C, and D) showing effects of $X_{1}, X_{2}$ and $X_{3}$ on $Y_{1}$ and $Y_{2}$ responses 
ed (0.643) $R^{2}$ values were in practical agreement. The amount of CMC $\left(X_{1}\right)$, SSG $\left(X_{2}\right)$ and starch $\left(X_{3}\right)$ have different effects on drug release kinetics from first order release $\left(\mathrm{Y}_{1}\right)$. In equation 11 the interaction of $X_{1}$ and $X_{2}, X_{1}$ and $X_{3}, X_{2}$ and $X_{3}$ were significant and showed positive effect on $\mathrm{Y}_{1}$ but not positive for $\mathrm{Y}_{2}$. The similar observations were reported by Sano et al., in 2013 using response surface methodology (12).

\section{$\boldsymbol{\beta}$-value of Weibull model}

Positive values of equation 12 showed optimization while negative values showed a negative effect between factors and responses. The model was found to be significant $(F$-value $=2.34 ; p<$ $0.001)$. The values for predicted $(0.654)$ and adjusted $(0.623) R^{2}$ values were in practical agreement. $R^{2}$ value was reasonably high for the $\beta$-value of Weibull model indicates a high degree of correlation between the experimental factors and response. In equation 12 the interaction of $X_{1}$ and $X_{2}, X_{1}$ and $X_{3}$, $\mathrm{X}_{2}$ and $\mathrm{X}_{3}$ were insignificant and showed a negative effect on $Y_{1}$ but showed positive effect for $Y_{2}$. Analysis of variance (ANOVA) of the response demonstrated that the quadratic model was significant $(p<0.001)$ and valid for the response. Solaiman et al., in 2016 studied the degree of relation between independent and dependent variables for the formulation of immediate-release tablets (13). Abbas et al., in 2017 studied the drug release pattern of pantoprazole by using box behnken response surface design (14).

\section{Experimental design validation}

The percent relative errors between the predicted and experimental values for each dependent variables were calculated and the values found to be within 5\%. The experimental values were in agreement with the predicted values confirming the predictability and validity of the responsive design. Acharya et al., in 2014 studied the validation of experimental design and reported the similar observation i.e. the relative standard was found to be within $5 \%$ (15).

\section{Pre-compression evaluation}

Angle of repose of powder blend was found to be $25.28 \pm 1.76^{\circ}$ to $28.34 \pm 1.53^{\circ}$ and in granules $26.39 \pm 1.230$ to $29.18 \pm 1.76$ showing good flow properties of all proposed formulations. Bulk density was ranged from $0.504 \pm 1.03$ to $0.508 \pm 1.23$ and $0.510 \pm 1.23$ to $0.512 \pm 1.09$ of powder blend and granules respectively while tapped density of powder blend was ranged between $0.542 \pm 1.23$ to 0.544 \pm 1.06 and granules were between $0.544 \pm 1.10$ to $0.546 \pm$ 1.04. Carr's index ranged from $7.142 \pm$ $0.51 \%$ to $8.139 \pm 0.43 \%$ and $7.549 \pm 0.47 \%$ to 8.436 $\pm 0.44 \%$ of the powder blend and granules respectively representing excellent compressibility of all planned formulations (16). Hausner's ratio of the
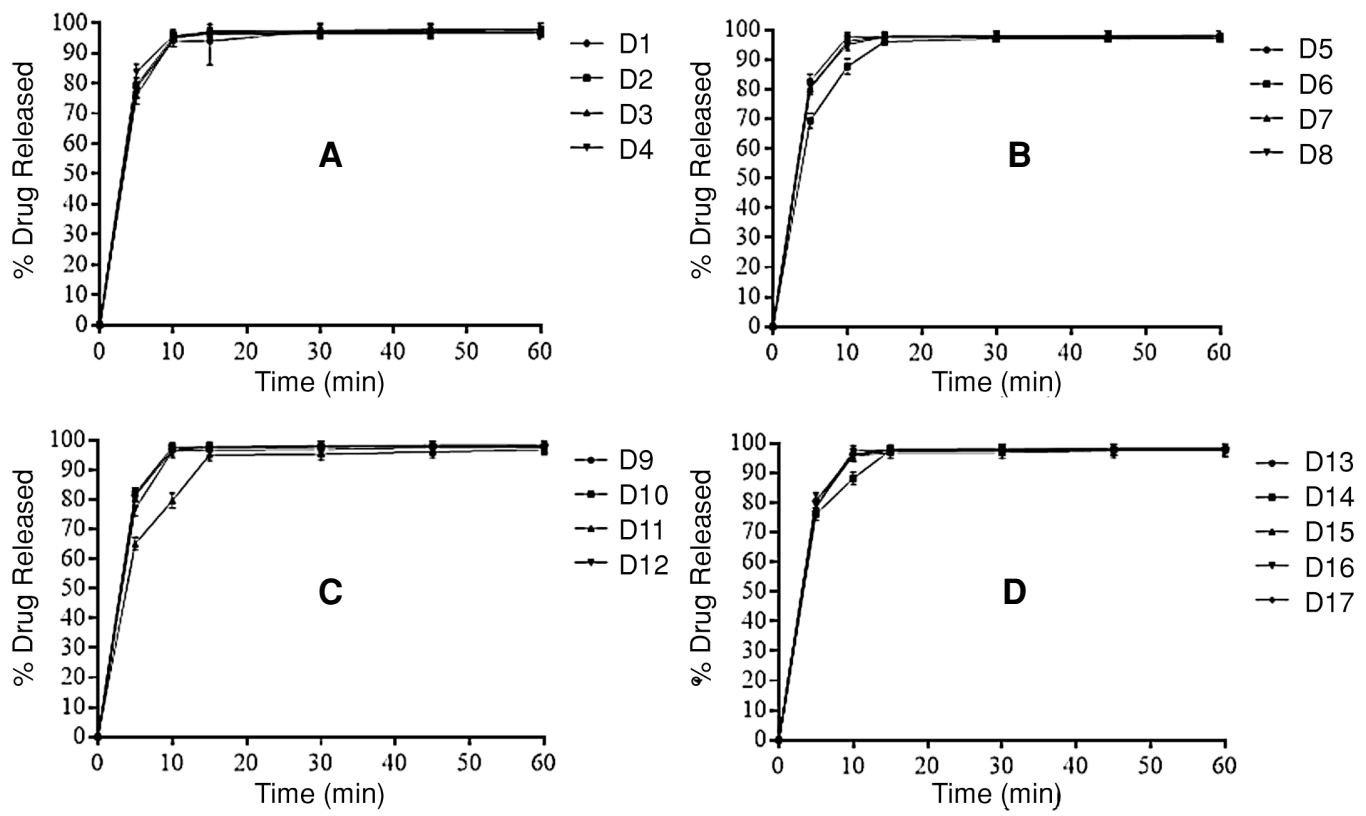

Figure 2. Release profile of DM from IR tablets. R1-R4 (A), R5-R8 (B), R9-R12 (C) and R13-R17 (D) (n=6) 


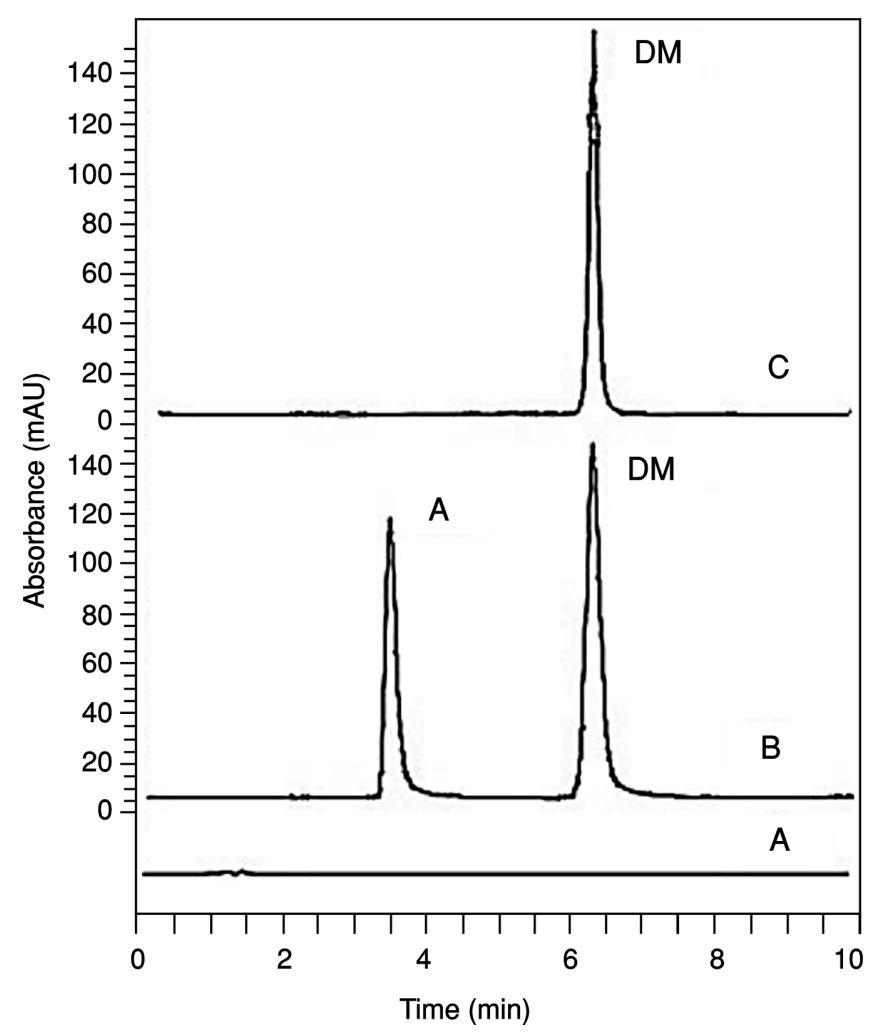

Figure 3. Chromatograms of blank solution (A), DM with IS (B) and DM (C) $(n=6)$

powder blend was 1.077 to 1.180 and granules were ranged from 1.110 to 1.23 showing better flow properties.

\section{Post-compression evaluation}

All prepared formulations were located within the acceptable range of weight variation, thickness, and diameter. Hardness' of tablets of all batches were found to vary from 2.125 to $3.347 \mathrm{~kg} / \mathrm{cm}^{2}$. Percentage friability of all formulations was less than $1 \%$ indicating good mechanical properties of tablets. Disintegration time of tablets decreased from 120 to $67 \mathrm{~s}$ by increasing the concentration of CMC and SSG from 1 to $4 \% \mathrm{w} / \mathrm{w}$ and 1 to $3 \% \mathrm{w} / \mathrm{w}$ respectively (17). It was observed that all formulations showed decrease in disintegration time due to the presence of superdisintegrants.

\section{In vitro drug release studies and release kinetics}

The in vitro drug release study was carried out in phosphate buffer $\mathrm{pH}$ 1.2. in order to mimic the conditions of gastrointestinal tract and results are shown in Figure 2. The results of the IR tablets (D1, D2, D7, D8 and D12) contained 2.5\% CMC, 2\% SSG and $22.5 \%$ starch showed that $80 \%$ of DM was released within 10 min and more than $90 \%$ of DM released was observed at $15 \mathrm{~min}$. Formulation D3 contained $2.5 \% \mathrm{CMC}, 1 \% \mathrm{SSG}$ and $15 \%$ starch showed $85 \%$ release was observed within $10 \mathrm{~min}$ and $90 \%$ percent DM was observed at $15 \mathrm{~min}$. The formulation D3 showed more dissolution pattern as compared to the D1, D2, D7, D8, and D12. The formulation D4 showed 80\% DM release was observed at $10 \mathrm{~min}$ because it contained $4 \% \mathrm{CMC}, 3 \% \mathrm{SSG}$ and $22.5 \%$ starch. The formulation D5 and D10 contained 4\% CMC and 2\% SSG 75\% DM release was observed within 10 minutes. The formulation D6, D11, D15 and D16 contained only 1\% CMC but having different concentrations of SSG and starch showed $80 \%$ drug release was observed within 10 min but more than $95 \%$ DM release was observed after $15 \mathrm{~min}$. This rapid release of DM was observed due to higher concentration of SSG but not due to the concentration of CMC. The formulation D9 contained $2.5 \% \mathrm{CMC}, 3 \% \mathrm{SSG}$ and $15 \%$ starch showed good release pattern of DM as shown in Figure 2(C) as compared to the formulation D17 which contained a similar concentration of CMC and SSG but different concentration of starch i.e. $30 \%$. This showed the effect of starch as a binder on the release 
profile of DM. The reported results are quite similar to the results of Ahmed et al., 2013 (18).

In kinetic release model $R^{2}$ values of first order release was ranged from 0.9959 to 0.9994 while in weibull model the value of $\beta$ ranged from 0.041 to 0.912 , which indicated the parabolic shape of drug release curve. Ranjha et al., in 2015 reported the similar observations of release kinetics of drugs (19). The $R^{2}$ values of all prepared formulations were nearest to the one which indicated first order release was followed by the drug through IR tablets. The value of $\beta$ of D6 formulation was 0.912 which was highest amongst all formulation but D10 formulation showed the minimum value i.e. 0.041 .

\section{Assay}

The DM was quantified by using developed HPLC method using isocratic mobile phase acetonitrile and phosphate buffer $\mathrm{pH} 4.0(50: 50 \mathrm{v} / \mathrm{v})$. The prepared sample was injected six times $(n=6)$ and

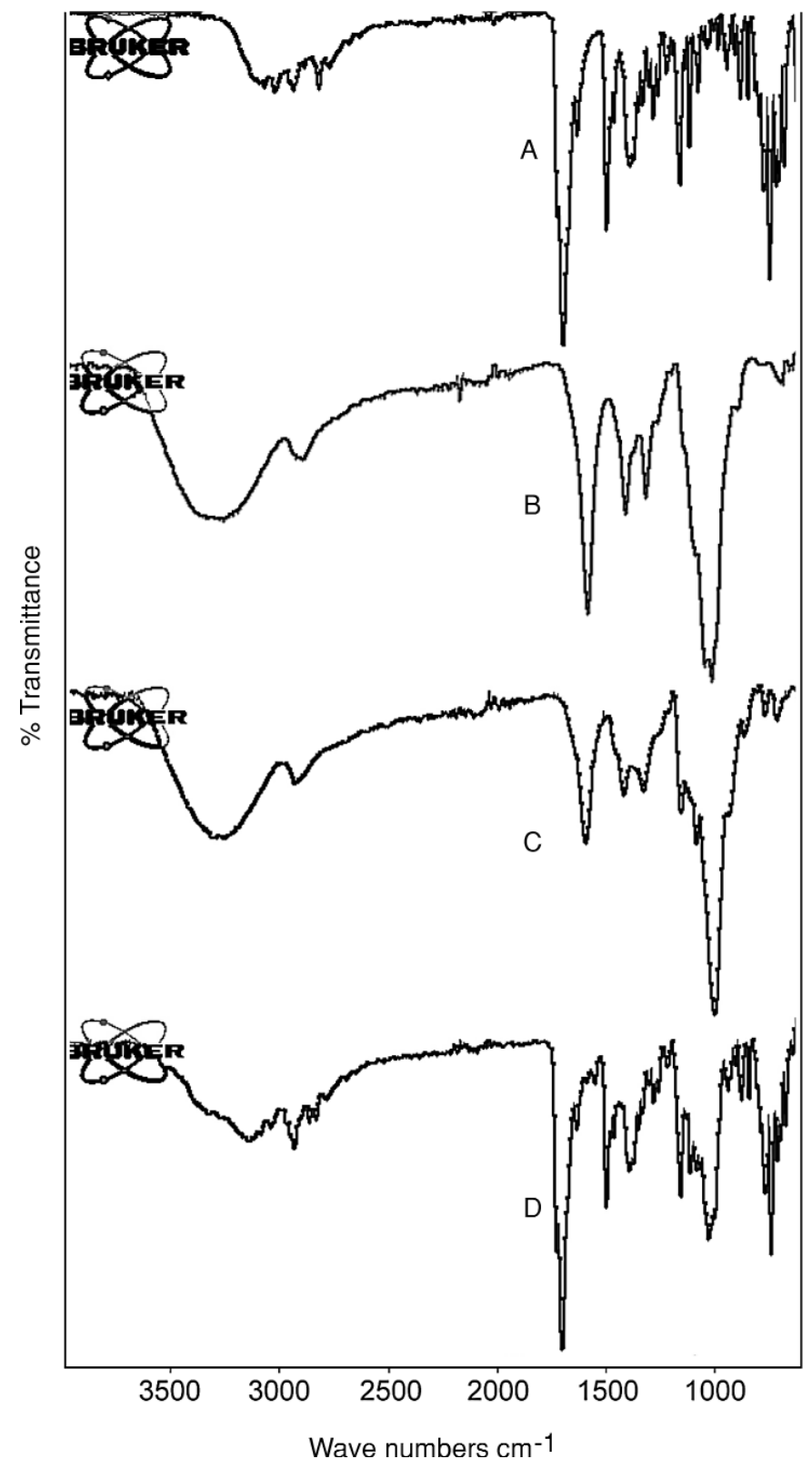

Figure 4. FTIR spectra of DM (A), CMC (B), SSG (C) and IR tablet of D9 (D) 


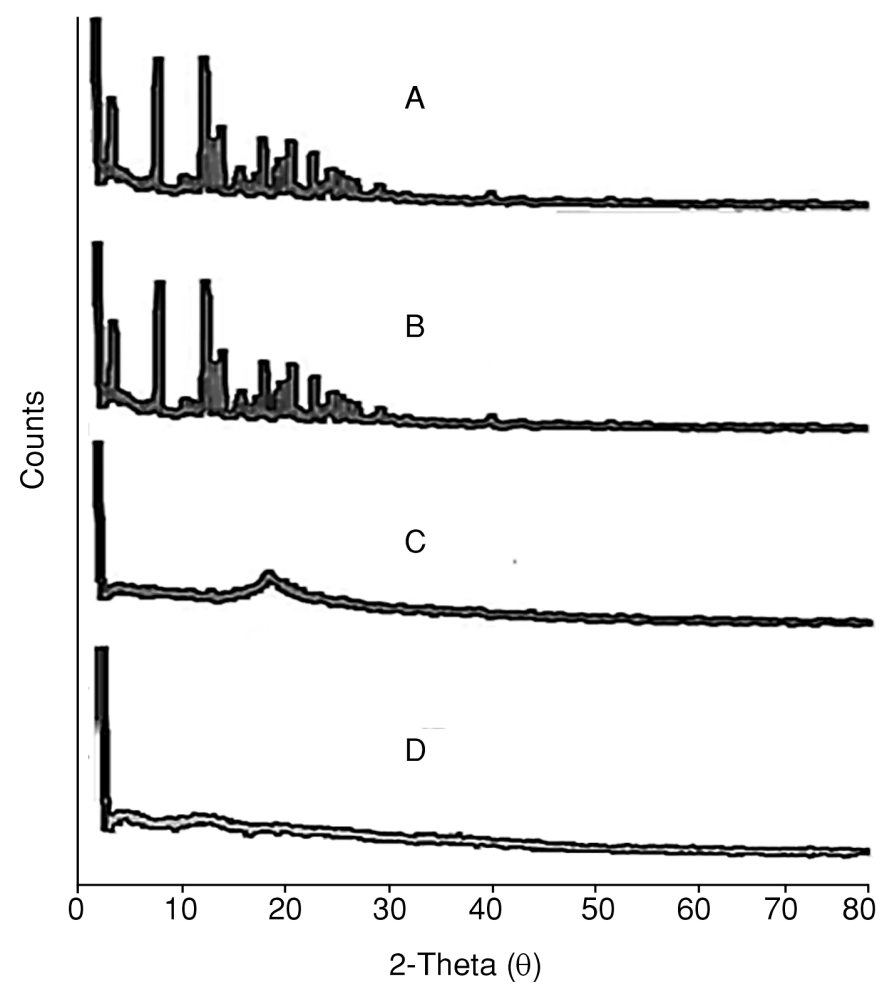

Figure 5. XRD diffractograms of DM (A), CMC (B), SSG (C) and IR tablet of D9 (D)

percentage recovery was found to be $99.89 \pm 0.5 \%$. This percentage recovery was within the official range of BP i.e. 85 to $115 \%$ and the \% RSD was $1.17 \%$ (20, 21). The chromatograms of blank solution, DM and DM with internal standard are shown in Figure 3.

\section{Fourier transform infrared spectroscopy (FTIR)}

Figure 4 showed the FTIR spectra of DM, CMC, SSG, and tablet of D9. DM showed features peaks at $3024.05 \mathrm{~cm}^{-1}$ due to $\mathrm{N}-\mathrm{H}$ stretching, $1687.40 \mathrm{~cm}^{-1}$ owing to $\mathrm{C}=0$ stretching and 1376.38 $\mathrm{cm}^{-1}$ due to $\mathrm{C}-\mathrm{H}$ bending (22). CMC showed peaks at $3245.67 \mathrm{~cm}^{-1}, 1585.40 \mathrm{~cm}^{-1}$ and $1019.27 \mathrm{~cm}^{-1}$ due to $-\mathrm{OH}$ stretching, carboxylic group stretching and stretching vibration of the $\mathrm{C}-\mathrm{OH}$ group respectively (23). Spectra of SSG showed peaks at $3253.00 \mathrm{~cm}^{-1}$ due to $-\mathrm{OH}$ stretching vibration and $1413.12 \mathrm{~cm}^{-1}$ owing to $-\mathrm{CH} 2$ symmetrical bond (23). Spectra of D9 IR tablet showed peaks at $2917.89 \mathrm{~cm}^{-1}, 1689.84$ $\mathrm{cm}^{-1}, 1383.06 \mathrm{~cm}^{-1} 1018.17 \mathrm{~cm}^{-1}$ due to $\mathrm{N}-\mathrm{H}$ and $\mathrm{C}=\mathrm{O}$ stretching of DM, C-H bending and stretching vibration of $\mathrm{C}-\mathrm{OH}$ group of $\mathrm{CMC}$ conclude the stable nature of IR tablets.

\section{X-ray diffractometry (XRD)}

The diffractograms of DM, CMC, SSG and tablet of D9 are shown in Figure 5. Diffraction lines of DM were observed at $2 \theta$ of $2^{\circ}, 4^{\circ}, 7^{\circ}, 12^{\circ}, 13^{\circ}$, $18^{\circ}, 20^{\circ}$, and $22^{\circ}$ due to its highly crystalline nature (24). CMC showed peaks at $4^{\circ}, 10^{\circ}, 16^{\circ}$ and $22^{\circ}$

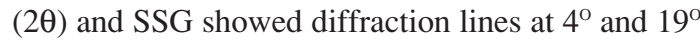

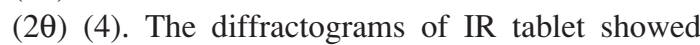
many characteristics peaks but disappearance of the peaks was observed in diffractograms of DM, CMC, and SSG. This indicates the considerable loss of crystallinity of drug and polymers in IR tablets.

\section{Differential scanning calorimetry (DSC)}

The thermograms of DM, CMC, SSG, and tablet of D9 are shown in Figure 6. Thermogram of DM showed an endothermic peak at $220^{\circ} \mathrm{C}$ which was the indication of a melting point of DM (24). CMC and SSG showed thermal behavior at $270^{\circ} \mathrm{C}$ and $240^{\circ} \mathrm{C}$. These results indicate that there was no noticeable interaction between drug and polymers.

\section{Stability studies and statistical analysis}

The stability studies of optimized formulation showed near proximity in hardness, friability, disintegration time and dissolution release profile (25). \% drug released showed $86 \%$ activity of IR tablets remained after 6 months and \% RSD was 5.671 as shown in Table 3. One-way ANOVA was applied 
Table 3. Results for stability study of immediate release tablets of D9 $(n=6)$

\begin{tabular}{|c|c|c|c|c|}
\hline Duration & Hardness $\left(\mathrm{kg} / \mathrm{cm}^{2}\right)$ & Friability $(\%)$ & Disintegration time $(\mathrm{s})$ & \% drug release \\
\hline 0 month & $2.925 \pm 0.078$ & $0.83 \pm 0.021$ & $74.2 \pm 0.50$ & $99.76 \pm 0.071$ \\
\hline 1 month & $2.913 \pm 0.056$ & $0.83 \pm 0.001$ & $75.3 \pm 0.70$ & $95.56 \pm 0.067$ \\
\hline 3 month & $2.629 \pm 0.178$ & $0.85 \pm 0.061$ & $77.6 \pm 0.40$ & $90.98 \pm 0.003$ \\
\hline 6 month & $2.507 \pm 0.052$ & $0.89 \pm 0.045$ & $80.2 \pm 0.50$ & $86.08 \pm 0.056$ \\
\hline Mean & $2.743 \pm 0.091$ & $0.85 \pm 0.032$ & $76.8 \pm 0.52$ & $92.25 \pm 5.315$ \\
\hline \%RSD & 3.317 & 3.764 & 0.677 & 5.761 \\
\hline
\end{tabular}

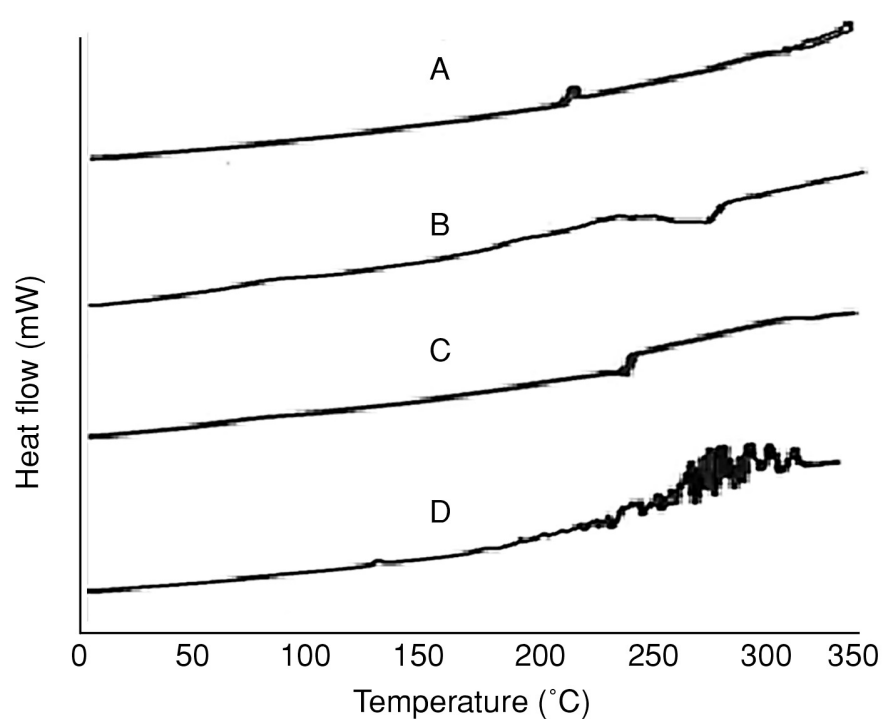

Figure 6. DSC thermograms of DM (A), CMC (B), SSG (C) and IR tablet of D9 (D)

on drug release data and value of $p$ was less than 0.05 and results were statistically significant.

\section{CONCLUSION}

This study discussed the positive application of response surface methodology for the development of IR tablets of DM. A systemic study using box behnken design revealed the most suitable content of CMC and SSG in the IR tablets. Optimized formulation fulfilled for the requirements of the target site. The IR tablets of DM were prepared successfully by using superdisintegrants such as CMC and SSG. The IR tablets were evaluated for various quality control parameters and the results were found within official limits. CMC and SSG were found to be the best in rapid disintegration and releasing of DM. The formulation D9 showing the best release and followed a first order release which confirmed the drug release dependent on the concentration. FTIR studies showed no chemical interaction between DM, super- disintegrants and other excipients. The present study clearly indicates the application of statistical optimization technique to predict the composition of formulation that gives optimum product parameters. The dosage form immediately releases the drug for rapid therapeutic effects. This dosage form holds an aptitude for further in vivo studies.

\section{REFERENCES}

1. Khan A., Iqbal Z., Khadra I., Ahmad L., Khan A. et al.: J. Pharm. Biomed. Anal. 121, 6 (2016).

2. Yoshizato T., Tsutsumi K., Kotegawa T., Imai H., Nakano S.: J. Chromatogr. B. 961, 86 (2014).

3. Lakshmi D.S., Trivedi N., Reddy C.: Carbohydr. Polym. 157, 1604 (2017).

4. Bhandari P.N., Jones D.D., Hanna M.A.: Ind. Crop. Prod. 41, 324 (2013).

5. Ölçer M., Ölçer A., İnce İ., Karasulu E.: Pharm. Dev. Technol. 20, 684 (2015). 
6. Elkhodairy K.A., Hassan M.A., Afifi S.A.: Saudi. Pharm. J. 22, 53 (2014).

7. Late S.G., Banga A.K.: Aaps. Pharmscitech. 11, 1627 (2010).

8. Ramteke K., Dighe P., Kharat A., Patil S.: Sch. Acad. J. Pharm. 3, 388 (2014).

9. Costa P., Lobo J.M.S.: Eur. J. Pharm. Sci. 13, 123 (2001).

10. Hanif M., Abbas G.: Adv. Polym Technol. (2017).

11. Abbas G., Hanif M.: J. App. Polym. Sci. 134, 44442 (2017).

12. Sano S., Iwao Y., Noguchi S., Kimura S., Itai S.: Int. J. Pharm. 448, 132 (2013).

13. Solaiman A., Suliman A.S., Shinde S., Naz S., Elkordy A.A.: Int. J. Pharm. 501, 87 (2016).

14. Abbas G., Hanif M., Khan M.A.: Des. Monomers. Polym. 20, 1 (2017).

15. Acharya S., Patra S., Pani N.R.: Carbohydr. Polym. 102, 360 (2014).

16. Paramakrishnan N., Jha S., Kumar K.J.: Int. J. Biolog. Macromol. 76, 320 (2015).
17. Hanif M., Shoaib M.H., Yousuf R.I., Sattar S., Nadeem M. et al.: Pak. J. Pharm. Sci. 27, (2014).

18. Ahmed I.S., Shamma R.N., Shoukri R.A.: Pharm. Dev. Technol. 18, 935 (2013).

19. Ranjha N.M., Hanif M., Afzal Z., Abbas G.: Pak. J. Pharm. Res. 1, 48 (2015).

20. Hanif M., Nazir N., Zia M.U., Chudhary B.A., Abbas G.: Latin. Americam. J. Pharm. 34, 1737 (2015).

21. Hanif M., Akhtar M.F., Naeem S., Wahid M., Shehzad M.A. et al.: Current. Pharm. Anal. 14, 3 (2018).

22. Osmani R.A.M., Aloorkar N.H., Thaware B.U., Kulkarni P.K., Moin A. et al.: Asian. J. Pharm. Sci. 10, 442 (2015).

23. Habibi N.: Spectrochim. Acta. Mol. Biomol. Spectrosc. 131, 55 (2014).

24. Nagarsenker M., Garad S., Ramprakash G.: J. Control. Rel. 63, 31 (2000).

25. Sarfraz R.M., Ahmad M., Mahmood A., Minhas M.U., Yaqoob A.: Trop. J. Pharm. Res.. 14, 1961 (2015).

Received: 21.07. 2017 